\title{
Carbon and glass hierarchical fibers : influence of carbon nanotubes on tensile, flexural and impact properties of short fiber reinforced composites
}

\begin{abstract}
Dense carbon nanotubes (CNTs) were grown uniformly on the surface of carbon fibers and glass fibers to create hierarchical fibers by use of floating catalyst chemical vapor deposition. Morphologies of the CNTs were investigated using scanning electronic microscope (SEM) and transmission electron microscope (TEM). Larger diameter dimension and distinct growing mechanism of nanotubes on glass fiber were revealed. Short carbon and glass fiber reinforced polypropylene composites were fabricated using the hierarchical fibers and compared with composites made using neat fibers. Tensile, flexural and impact properties of the composites were measured, which showed evident enhancement in all mechanical properties compared to neat short fiber composites. SEM micrographs of composite fracture surface demonstrated improved adhesion between CNT-coated fiber and the matrix. The enhanced mechanical properties of short fiber composites was attributed to the synergistic effects of CNTs in improving fiber-matrix interfacial properties as well as the CNTs acting as supplemental reinforcement in short fiber-composites.
\end{abstract}

Keyword: Nanocomposites; Carbon nanotubes; Vapor deposition; Impact 